\title{
On American Options Under the Variance Gamma Process
}

\author{
ARIEL ALMENDRAL* \& CORNELIS W. OOSTERLEE** \\ *Norwegian Computing Center, Blindern, Oslo, Norway. Currently at Delft University of Technology, \\ **Delft University of Technology, Delft Institute of Applied Mathematics (DIAM), The Netherlands
}

(Received 9 February 2005; in revised form 13 March 2006)

ABSTRACT American options are considered in a market where the underlying asset follows a Variance Gamma process. A sufficient condition is given for the failure of the smooth fit principle for finite horizon call options. A second-order accurate finite-difference method is proposed to find the American option price and the exercise boundary. The problem is formulated as a Linear Complementarity Problem and solved numerically by a convenient splitting. Computations have been accelerated with the help of the Fast Fourier Transform. A stability analysis shows that the scheme is conditionally stable, with a mild stability condition of the form $\mathrm{k}=\mathrm{O}\left(|\log (\mathrm{h})|^{-1}\right)$. The theoretical results are verified numerically throughout a series of numerical experiments.

KEY WoRDS: Integro-differential equations, variance gamma, finite differences, FFT

\section{Introduction}

The variance gamma (VG) process was first introduced in financial modelling by Madan and Seneta (1990) to cope with the shortcomings of the Black-Scholes model. Empirical studies of financial time series have revealed that the normality assumption in the Black-Scholes theory cannot capture heavy tails and asymmetries present in the empirical log-returns. The empirical densities are usually too peaked compared to the normal density; a phenomenon known as excess of kurtosis. In addition, the Black-Scholes assumption on constant parameters is inconsistent since, for example, a numerical inversion of the Black-Scholes equation based on market prices from different strikes and fixed maturity, produces a so-called volatility skew or smile. In these aspects, VG modelling is superior to the Black-Scholes model: on one hand, it has the property that daily log-returns have heavy tails and on the other hand, for longer periods it approaches normality, which is also consistent with empirical studies. Moreover, by introducing extra parameters, one can control the

Correspondence Address: A. Almendral, Norwegian Computing Center, Gaustadalleen 23, Postbox 114, Blindern, N-0314, Oslo, Norway. Email: Ariel.Almendral.Vazquez@nr.no. Now at Delft University of Technology. 
kurtosis and asymmetry of the log-return density, and one is also able to fit the smile in the implied volatility (Madan and Seneta, 1990; Carr et al., 1998).

There exist, however, important drawbacks when modelling with the VG process. For example, a hedging strategy for the writer of the option that will completely remove the risk of writing the option does not exist, in general; in other words, a portfolio that replicates any contingent claim cannot be constructed.

In this paper we give a sufficient condition for which the so-called smooth fit principle for American options under the VG process does not hold. For the infinite horizon case it has been proved by Alili and Kyprianou (2005). Secondly, we propose a tractable numerical method based on a linear complementarity formulation of the free boundary value problem for the $\mathrm{VG}$ prices. A numerical valuation of $\mathrm{VG}$ American options was carried out by Hirsa and Madan (2004), using finite differences on a non-linear interpretation of the PIDE. Compared to Hirsa and Madan (2004), the method proposed here is more easily extendible to other finite variation processes. Moreover, the method presented can naturally handle the asymptotic behaviour of the free boundary near expiry. We accompany the method by a stability analysis, similar in spirit to the Von Neumann stability analysis given by Andersen and Andreasen (2000) for the jump-diffusion case.

Another general model based on the Carr-Geman-Madan-Yor (CGMY) process, introduced by Koponen (1995), is numerically solved by Matache et al. (2003), by a combination of variational inequalities and the Galerkin method, with a convenient wavelet basis to compress the resulting full matrix. In the present paper, however, a simpler implicit-explicit method is proposed, which, in combination with a fast convolution procedure based on the fast Fourier transform, offers an effective pricing procedure for European and American vanilla options. In a previous paper (Almendral and Oosterlee, 2005), we used matrix splitting to solve numerically jump-diffusion European vanilla options; see also D'Halluin et al. (2005), where the differential operator is handled implicitly, and D'Halluin et al. (2004) for an application of the penalty method to American options under Merton's model.

The outline of the paper is as follows. In the following section we offer a brief introduction into the VG market model and the option pricing problem, and discuss the failure of the smooth-fit principle. The next two sections are dedicated to the numerical valuation of a VG call option. In the third section we reformulate the problem as a linear complementarity problem and consider a timestepping method to solve it numerically. In the fourth section a stability analysis is carried out to get the insight that the scheme is conditionally stable, with a mild stability restriction, and in the fifth section we show numerical experiments that confirm the theoretical findings. The sixth section concludes.

\section{A Market Modelled by the Variance Gamma Process}

In this section we summarize known facts about a market driven by the VG process (Carr et al., 1998). The VG process is obtained by evaluating a drifted Brownian motion at random times given by a gamma process. For a definition of the gamma process see Cont and Tankov (2004). The three parameters determining the VG process $X(t ; \sigma, v, \theta)$ are the volatility $\sigma$ of the Brownian motion, the variance $v$ of the 
gamma distributed time and the drift $\theta$ of the time-changed Brownian motion with drift.

Consider a market consisting of one bank account $B(t)$, with risk-free interest rate $r$, and some risky asset $S(t)$. The bank account evolves as usual according to the law $\mathrm{d} B(t)=r B(t) \mathrm{d} t$ and the asset $\{S(t)\}_{t \geqslant 0}$ follows the exponential dynamics:

$$
S(t)=S_{0} \exp (L(t))
$$

where

$$
L(t)=-\alpha t+X(t ; \sigma, v, \theta)
$$

Here $-\alpha$ is the drift of the logarithmic price of the asset. We assume also that the asset pays its owner a continuous dividend $q \geqslant 0$. The process $\{L(t)\}_{t \geqslant 0}$ is a so-called Lévy process, i.e. a process with stationary, independent increments.

Assume the existence of some equivalent martingale measure (EMM) $Q$ such that the discounted process $\left\{e^{-(r-q) t} S(t)\right\}_{t \geqslant 0}$ becomes a martingale, and suppose that the parameters $\sigma, v$ and $\theta$ are also chosen to be risk-neutral. For a discussion of the issue of choosing $Q$ see Cont and Tankov (2004).

We may write the Lévy-Khintchine representation of $L(t)$ with respect to this new measure as follows:

$$
E_{Q}\left(e^{i z L(t)}\right)=\exp \left[t\left(-i \alpha z+\int_{\mathbb{R}}\left(e^{i z x}-1\right) k(x) \mathrm{d} x\right)\right]
$$

where $k(x)$ is known as the Lévy density.

In a risk-neutral world, it is possible to find the form of the drift $\alpha$. Namely, by substituting $z=-i$ in (3), and comparing the result with the so-called risk-neutrality condition

$$
E_{Q}[S(t)]=S_{0} e^{t(r-q)}
$$

where $r$ and $q$ are the risk-free interest rate and the dividend paid by the asset, respectively, one arrives at

$$
\alpha=q-r-\omega
$$

where $\omega$ is some 'compensation constant' given by

$$
\omega=\int_{\mathbb{R}}\left(1-e^{y}\right) k(y) \mathrm{d} y
$$

Notice that the notation used here is as in Hirsa and Madan (2004) (the solution method however will be completely different, as mentioned in the introduction). It is possible to compute $\omega$ by using the characteristic function of the process $\{X(t)\}_{t \geqslant 0}$ (Carr et al., 1998):

$$
E_{Q}\left(e^{i z X(t)}\right)=\left(1-i z \theta v+z^{2} \sigma^{2} v / 2\right)^{-t / v}
$$

Substituting $z=-i$ in this expression and using the risk-neutrality condition, one 
finds the following form for $\omega$ :

$$
\omega=\frac{1}{v} \ln \left(1-\theta v-\sigma^{2} v / 2\right)
$$

It is also known that the Lévy density $k(x)$ has the form:

$$
k(x)=\left\{\begin{array}{lll}
\frac{1}{v} \frac{\exp \left(-\lambda_{+}|x|\right)}{|x|} & \text { if } & x>0 \\
\frac{1}{v} \frac{\exp \left(-\lambda_{-}|x|\right)}{|x|} & \text { if } & x<0
\end{array}\right.
$$

where the positive parameters $\lambda_{ \pm}$are given by

$$
\lambda_{ \pm}^{-1}=\sqrt{\frac{\theta^{2} v^{2}}{4}+\frac{\sigma^{2} v}{2}} \pm \frac{\theta v}{2}
$$

Note that the positive exponent $\lambda_{+}$must be larger than 1 for the constant $\omega$ to be well defined.

\section{American Options in a VG Market}

Consider an American call option on the underlying $S(t)$, with expiry $T$, and strike price $K$. For the non-degenerate case, i.e. for infinite activity jump processes combined with a diffusion process, it is shown in Bensoussan and Lions (1984) that the price $v(t, s)$ solves a certain set of integro-differential inequalities. A discussion of the degenerate case (purely jump processes, like the VG process) for European options is provided in Cont and Voltchkova (2005), where the concept of viscosity solution is used to obtain existence and uniqueness results under rather weak conditions. For the degenerate case a classical solution is not appropriate due to general lack of regularity. For the American case, Theorem 6.1 in Boyarchenko and Levendorskii (2002) gives conditions under which the stochastic representation is a continuous solution of these integro-differential inequalities. It is beyond the scope of this paper to discuss existence and uniqueness results for these equations. We state the equations for a call, and assume that the stochastic representation is a continuous solution.

Let $t$ stand for time to maturity. Let the continuation region consist of intervals of the form $(0, c(t))$, for a certain increasing function $c(t)$, not known a priori. The continuation region has this special form if the solution is convex, non-decreasing, and satisfies the Lipschitz condition; see Lemma 2.1. The free boundary value problem for the American option price is the following:

$$
\begin{aligned}
& v_{t}+(q-r-\omega) s v_{s}+r v \\
& -\int_{\mathbb{R}}\left(v\left(t, s e^{y}\right)-v(t, s)\right) k(y) d y=0 \quad \text { for } t \in(0, T], \quad s \in(0, c(t))
\end{aligned}
$$

with $k(y)$ as in (9) and initial and boundary conditions

$$
\begin{gathered}
v(0, s)=(s-K)^{+} \quad \text { for } s \geq 0 \\
v(t, 0)=0 \quad \text { for } t \in[0, T]
\end{gathered}
$$




$$
v(t, c(t))=c(t)-K \quad \text { for } t \in(0, T]
$$

The formulation of (11) is already stated forward in time. Two extra conditions on the solution must be imposed:

$$
\begin{aligned}
& v_{t}+(q-r-\omega) s v_{s}+r v \\
& -\int_{\mathbb{R}}\left(v\left(t, s e^{y}\right)-v(t, s)\right) k(y) d y \geq 0 \quad \text { for } t \in(0, T], \quad s \in(c(t), \infty)
\end{aligned}
$$

and

$$
v(t, s) \geq(s-K)^{+} \quad \text { for } t \in(0, T], s \in \mathrm{R}^{+}
$$

Condition (15) states that the integro-differential operator is constant in sign on the exercise region. This is an important remark when reformulating this problem as a linear complementarity problem. The second condition (16) has a parallel in obstacle problems, where the obstacle in this case is the payoff function.

\section{The Smooth Fit Principle}

The smooth fit principle (or smooth paste principle) was first introduced in the financial literature by Samuelson (1965) under the name 'high contact-condition'. The principle essentially states that the derivative of the Black-Scholes American option price is a continuous function, also at the exercise boundary. The geometrical interpretation is that, for each fixed $t>0$, the function $v(t, s)$ as a function of the asset value $s$, smoothly enters into the payoff function, in the Black-Scholes world.

We will make use of the following monotonicity result.

Lemma 2.1. The mappings $t \mapsto v(t, s), \quad s \mapsto v(t, s)$ and $s \mapsto v(t, s)-s$ are nondecreasing, nondecreasing and non-increasing respectively.

Proof. The proof is the same as in Karatzas and Shreve (1998), Lemma 7.4. We include it here for the sake of completeness. The first assertion follows from the fact that a stopping time in $[0, t]$ is also a stopping time in $\left[0, t^{\prime}\right]$, for $t \leqslant t^{\prime}$. The second assertion is also immediate since the function $s \mapsto(s H(\tau)-K)^{+}$is nondecreasing. Here, $H(t)$ is the price process starting at 1 , or, in other words, $S(t)=s H(t)$, with

$$
H(t):=\exp [(r-q) t+\omega t+X(t)]
$$

To prove the third assertion, let $0 \leqslant s_{1}<s_{2}<\infty$ and let $\tau_{2}$ be some optimal stopping time corresponding to $s_{2}$. Then

$$
\begin{aligned}
v\left(t, s_{2}\right)-v\left(t, s_{1}\right) & =E_{Q}\left[e^{-r \tau_{2}}\left(s_{2} H\left(\tau_{2}\right)-K\right)^{+}\right]-v\left(t, s_{1}\right) \\
& \leq E_{Q}\left[e^{-r \tau_{2}}\left\{\left(s_{2} H\left(\tau_{2}\right)-K\right)^{+}-\left(s_{1} H\left(\tau_{2}\right)-K\right)^{+}\right\}\right] \\
& \leq\left(s_{2}-s_{1}\right) E_{Q}\left[e^{-r \tau_{2}} H\left(\tau_{2}\right)\right]
\end{aligned}
$$

where we have used the inequality $a^{+}-b^{+} \leqslant(a-b)^{+}$, valid for any $a, b \in \mathrm{R}$. It remains 
to observe that, since $\left\{e^{-(r-q) t} H(t)\right\}_{t \geqslant 0}$ is a $Q$-martingale, then $\left\{e^{-r t} H(t)\right\}_{t \geqslant 0}$ is a supermartingale. Hence, by Doob's Optional Sampling Theorem, $E_{Q}\left[e^{-r \tau 2} H\left(\tau_{2}\right)\right] \leqslant 1$.

We will now show that the smooth-fit principle is not valid, if we assume that the dividend $q$ satisfies a certain condition. To write down this condition, let us recall the expression for $\omega$ :

$$
\omega=\int_{\mathbb{R}}\left(1-e^{y}\right) k(y) \mathrm{d} y
$$

If we denote by $\omega_{-}$and $\omega_{+}$the integral above over the negative semi-axis and the positive semi-axis, respectively, then we may split $\omega$ as $\omega=\omega_{-}+\omega_{+}$.

We show next that if

$$
q>r+\omega_{-}
$$

the derivative of a call option turns out to be discontinuous. An important issue should be discussed at this point. We do not mention in which sense $v$ is a solution to (11)-(16). Solutions in the viscosity sense or in the weak sense usually help giving a proper meaning to the concept of solution. However, if we know that $v$ is a continuously increasing function in $s$, then it is well known that the derivatives exist almost everywhere. Hence, for $s$ in the continuation region, the following inequality always makes sense (at least a.e.):

$$
(q-r-\omega) s v_{s}+r v-\int_{\mathbb{R}}\left(v\left(t, s e^{y}\right)-v(t, s)\right) k(y) \mathrm{d} y \leq 0
$$

Let us rewrite this expression as

$$
(q-r-\omega) s\left(1-v_{s}\right) \geq(q-r-\omega) s+r v-\int_{\mathbb{R}}\left(v\left(t, s e^{y}\right)-v(t, s)\right) k(y) \mathrm{d} y
$$

Now we use some properties that we require from the solution, i.e. for $y<0$, $v\left(t, s e^{y}\right)-v(t, s) \leqslant 0$, and $v \geqslant s-K$. These inequalities lead us to the following estimate

$$
(q-r-\omega) s\left(1-v_{s}\right) \geq q s-\omega s-r K-\int_{0}^{\infty}\left(v\left(t, s e^{y}\right)-v(t, s)\right) k(y) \mathrm{d} y
$$

The Lipschitz property is now invoked. As proved in Lemma 2.1, given that $s e^{y}>s$, for $y>0$, one has

$$
v\left(t, s e^{y}\right)-v(t, s) \leq s\left(e^{y}-1\right)
$$

so that the right-hand side in (20) may be bounded from below by $q s-\omega_{-} s-r K$, which for $s \geqslant K$ gives

$$
(q-r-\omega) s\left(1-v_{s}\right) \geq K\left(q-r-\omega_{-}\right)
$$

If we also know that the free boundary is an increasing function, we arrive at the following positive lower bound

$$
1-v_{s} \geq \frac{K}{c(T)} \frac{q-r-\omega_{-}}{q-r-\omega}>0, \quad \forall t \in(0, T) \text { and } \forall s \in[K, c(t))
$$

Roughly speaking, if we want a solution satisfying the usual properties of an 
American option price, this solution cannot have a continuous derivative and at the same time satisfy the integro-differential equations.

Remark 2.1. The condition in Alili and Kyprianou (2005) for the lack of smoothfit in the perpetual case of a call option is that the drift of the Lévy process has to be negative, i.e. $q-r-\omega>0$. Note that, since $\omega_{+}$is non-negative, $q-r-\omega_{-} \geqslant q-r-\omega>0$, that is, (17) implies negative drift.

\section{Numerical Valuation of the American VG Price}

Our goal here is to solve the free boundary problem (11)-(16) numerically, when the asset pays a positive dividend.

We are interested in the effect of adding a diffusion part to the VG process: The new coefficient will then be denoted by $\bar{\sigma}$. This parameter is later used to compare numerically the regularity of the free boundary with diffusion (Generalized VG process, $\bar{\sigma}>0$ ) and without diffusion (VG process, $\bar{\sigma}=0$ ). The omission of this parameter does not really affect the numerical method that we are about to explain.

We will not work directly on (11)-(16), but rather on its logarithmic version, i.e. we change variables to $x=\ln s$ and solve for the new function

$$
u(t, x):=v\left(t, e^{x}\right)
$$

To transform equations (11)-(16) to these new variables, it is also convenient to define the 'logarithmic continuation region':

$$
\tilde{\mathcal{C}}=\left\{(t, x) \in(0, \infty) \times \mathbb{R} \mid u(t, x)>\left(e^{x}-K\right)^{+}\right\}
$$

and the optimal logarithmic asset value at which the option should be exercised:

$$
\tilde{c}(t)=\sup \left\{x \in \mathbb{R} \mid u(t, x)>\left(e^{x}-K\right)^{+}\right\}, \quad t \in(0, \infty)
$$

We are ready now to present the formulation of (11)-(16) in the logarithmic price:

$$
\begin{gathered}
u_{t}-\mathcal{L} u=0, \quad t>0, \quad x<\widetilde{c}(t) \\
u(t, x)=e^{x}-K, \quad t>0, \quad x \geq \widetilde{c}(t) \\
u(t, x) \geq\left(e^{x}-K\right)^{+}, \quad t>0, \quad x \in \mathbb{R} \\
u_{t}-\mathcal{L} u \geq 0, \quad t>0, \quad x>\widetilde{c}(t) \\
u(0, x)=\left(e^{x}-K\right)^{+}, x \in \mathbb{R}
\end{gathered}
$$

where the operator $\mathcal{L}$ is defined in the following way:

$$
\begin{aligned}
& \mathcal{L} \varphi:=\frac{\bar{\sigma}^{2}}{2} \varphi_{x x}-\left(q-r+\frac{\bar{\sigma}^{2}}{2}\right) \varphi_{x}-r \varphi \\
& +\int_{\mathbb{R}}\left[\varphi(t, x+y)-\varphi(t, x)-\left(e^{y}-1\right) \varphi_{x}(t, x)\right] k(y) \mathrm{d} y
\end{aligned}
$$

Note that we have included a second-order term. 
This problem may be cast as the following so-called linear complementarity problem

$$
\begin{cases}u_{t}-\mathcal{L} u \geq 0 & \text { in }(0, T] \times \mathbb{R} \\ u \geq \psi & \text { in }[0, T] \times \mathbb{R} \\ \left(u_{t}-\mathcal{L} u\right)(u-\psi)=0 & \text { in }(0, T] \times \mathbb{R} \\ u(0, x)=\psi(x) & \end{cases}
$$

where the initial condition is given by

$$
\psi(x):=\left(e^{x}-K\right)^{+}
$$

This formulation of the problem is the basis for the numerical method presented.

\section{Discretization}

We discretize the linear complementarity problem (33) by finite differences. The idea of the method is to consider one part of the integral term implicitly and the remaining explicitly. The implicit part will make sure that the scheme requires only a few time-steps. We will discuss the stability in a later section.

Consider a computational domain of the form $[0, T] \times\left[x_{\min }, x_{\max }\right]$. Let the time interval $[0, T]$ be divided into $M$ equal parts: $0=t_{0}<t_{1}<\ldots<t_{M}=T$, with $t_{m}=m k$, $m=0,1, \ldots, M$ and $k=T / M$. The spatial interval $\left[x_{\min }, x_{\max }\right]$ contains the point $\ln K$, and $x_{\min }=x_{0}<x_{1}<\ldots<x_{N}=x_{\max }$, with $x_{n}=x_{\min }+n h, n=0, \ldots, N$, and $h$ is such that $h=\left(x_{\max }-x_{\min }\right) / N$.

We split the operator $\mathcal{L}$ into a sum of two operators $\mathcal{A}$ and $\mathcal{B}$ :

$$
\begin{aligned}
\mathcal{A} \varphi:=\frac{\bar{\sigma}^{2}}{2} \varphi_{x x} & -\left(q-r+\frac{\bar{\sigma}^{2}}{2}-\omega(h)\right) \varphi_{x}-r \varphi \\
+ & \int_{|y| \leq h}\left[\varphi(t, x+y)-\varphi(t, x)-\left(e^{y}-1\right) \varphi_{x}(t, x)\right] k(y) \mathrm{d} y
\end{aligned}
$$

where we introduce the notation:

$$
\omega(h)=\int_{|y| \geq h}\left(1-e^{y}\right) k(y) \mathrm{d} y
$$

and

$$
\mathcal{B} \varphi:=\int_{|y| \geq h}[\varphi(t, x+y)-\varphi(t, x)] k(y) \mathrm{d} y
$$

Now, define the time approximations $u^{m} \approx u\left(t_{m}, x\right)$ and consider the following 
implicit-explicit timestepping method to solve (33):

$$
\left\{\begin{array}{l}
\frac{u^{m+1}-u^{m}}{k}-\mathcal{A} u^{m+1}-\mathcal{B} u^{m} \geq 0 \\
u^{m+1} \geq \psi \\
\left(\frac{u^{m+1}-u^{m}}{k}-\mathcal{A} u^{m+1}-\mathcal{B} u^{m}\right)\left(u^{m+1}-\psi\right)=0 \\
u^{0}=\psi
\end{array}\right.
$$

This method is related to Zhang (1997) for the computation of the American put for Merton's model.

Spatial discretization of $\mathcal{A}$. The idea here is to approximate all the integrals to $O\left(h^{2}\right)$, in the presence of the singular density,

$$
k(y)=O\left(\frac{1}{y}\right), y \rightarrow 0
$$

The trapezoidal rule applied to the integral operator gives, for the positive interval

$$
\begin{aligned}
& \int_{0}^{h}\left[w\left(x_{n}+y\right)-w\left(x_{n}\right)-\left(e^{y}-1\right) w_{x}\left(x_{n}\right)\right] k(y) \mathrm{d} y \\
& =\int_{0}^{h}\left[w\left(x_{n}+y\right)-w\left(x_{n}\right)-y w_{x}\left(x_{n}\right)-\left(e^{y}-1-y\right) w_{x}\left(x_{n}\right)\right] k(y) \mathrm{d} y \\
& =\frac{\delta_{2}(w)}{2} \int_{0}^{h} y^{2} k(y) \mathrm{d} y-\frac{\delta_{1}(w)}{2} \int_{0}^{h} y^{2} k(y) \mathrm{d} y+O\left(h^{2}\right)
\end{aligned}
$$

where we write

$$
\delta_{1}(w):=\frac{w_{i+1}-w_{i-1}}{2 h}, \quad \delta_{2}(w):=\frac{w_{i+1}-2 w_{i}+w_{i-1}}{h^{2}}
$$

The term $e^{y}-1-y$ has been substituted by $y^{2} / 2$ with an error of the order $O\left(y^{3}\right)$. We have carried out some approximations of order $O\left(h^{2}\right)$, and the terms $\int_{0}^{h} y^{2} k(y) \mathrm{d} y$ are of the same order, according to (39). Therefore, dropping the term

$$
\int_{0}^{h}\left[w\left(x_{n}+y\right)-w\left(x_{n}\right)-\left(e^{y}-1\right) w_{x}\left(x_{n}\right)\right] k(y) \mathrm{d} y
$$

does not affect the accuracy of the scheme. Numerical results confirm this fact.

Remark 3.1. The discretization order for the integrand $(w(x+y)-w(x)) k(y)$ by the trapezoidal method deteriorates as $k(y)=O\left(y^{-1-\rho}\right), \rho>0$ since the integrand becomes less smooth. The same algorithm has been tested for the CGMY process in Almendral (2005) where we found only first-order spatial convergence. A secondorder accurate approximation for the CGMY process, based on an integration-byparts technique, is part of a forthcoming report.

In this paper, the convection term is discretized by a Lax-Wendroff scheme or by the central scheme. The reason for choosing a Lax-Wendroff scheme is that we 
would like to take a closer look at the smooth-fit principle in the numerical examples below. It is well known that the central scheme is not monotone, with incorrect oscillations occurring in the solution in the vicinity of jumps. The Lax-Wendroff scheme is not monotone either, but for the examples in this paper, where the solution developes a kink at exercise, the Lax-Wendroff scheme is much better behaved than the central scheme.

We recall that the Lax-Wendroff discretization (in this case implicit) of a term $Q w_{x}$ is the following (LeVeque, 1990; Strikwerda, 1989):

$$
Q \frac{w_{n+1}^{m}-w_{n-1}^{m}}{2 h}+Q^{2} \frac{k}{h^{2}}\left(w_{n+1}^{m}-2 w_{n}^{m}+w_{n-1}^{m}\right)
$$

The first term is a plain central scheme, and the second one is the Lax-Wendroff update. This discretization is second-order accurate in space. With discretization schemes based on total variation diminishing (TVD) or essentially nonoscillatory (ENO) (see LeVeque, 1990) stability concepts wiggles can be avoided at any time, but these concepts are somewhat involved and beyond the scope of the present paper.

Spatial discretization of $\mathcal{B}$. Away from the origin, the integral term in $\mathcal{B}$ may be split into a sum of two terms:

$$
\int_{|y| \geq h}\left[w\left(x_{n}+y\right)-w\left(x_{n}\right)\right] k(y) \mathrm{d} y=J_{n}-w_{n} \lambda(h)
$$

with the obvious notation

$$
\begin{gathered}
J_{n}=\int_{|y| \geq h} w\left(x_{n}+y\right) k(y) \mathrm{d} y \\
\lambda(h)=\int_{|y| \geq h} k(y) \mathrm{d} y
\end{gathered}
$$

The most expensive part lies in computation of the numbers $J_{n}$. Let us explain first how these entries may be computed approximately. Later we give a method to accelerate the resulting correlations. It is assumed here that the number of spatial points $N$ is an even number.

Let $L$ be an integer larger than 1 . The trapezoidal rule on truncation of the integral gives:

$$
\begin{aligned}
J_{n}=\int_{|y| \geq h} w\left(x_{n}+y\right) k(y) \mathrm{d} y & \approx \int_{h \leq|y| \leq L h} w\left(x_{n}+y\right) k(y) \mathrm{d} y \\
& =h \sum_{l=-L}^{L} w_{n+l} k_{l} \rho_{l}+O\left(h^{2}\right), \quad n=0,1, \ldots, N
\end{aligned}
$$

The following notation was employed:

$$
k_{l}=k(l h), \quad l \neq 0
$$




$$
\rho_{l}= \begin{cases}1 / 2 & \text { if } l \in\{-L,-1,1, L\} \\ 1 & \text { otherwise }\end{cases}
$$

and for $m=0$ we have redefined $k(0)$ as 0 . Error estimates for this truncation are given in Cont and Voltchkova (2005).

We need to do something special for indices $n+l$ such that $x_{n+l} \leqslant x_{\text {min }}$ (i.e. $n+l \leqslant 0$ ) or $x_{n+l} \geqslant x_{\max }$ (i.e. $n+l \geqslant N$ ). In the case of an American option we simply let $w_{n+l}:=\psi_{n+l}$. In other words, we substitute $w$ by the payoff function for points lying outside the computational domain. For European options the situation is different since the asymptotic behaviour of a call or a put depends on the interest rate $r$. For example, a call approaches $e^{x}-K e^{-r t}$ for $x$ large, and 0 for $x$ large negative. In the case of a put we have $K e^{-r t}$ for $x$ large negative and 0 for large positive. Another possibility to treat the negative far field is to consider the ODE that results from the substitution $s=0$ (the same as $x \rightarrow-\infty$ ) in the PIDE, that is, $u_{t}=-r u$. The solution of this ODE is of the form $C e^{-r t}$, and the constant $C$ may be determined from previous values $u\left(x_{\min }\right)$.

For the coefficients $\lambda(h)$ and $\omega(h)$ we may use the same approximation. For example

$$
\lambda(h)=\int_{|y| \geq h} k(y) \mathrm{d} y \approx h \sum_{l=-L}^{L} k_{l} \rho_{l}
$$

In our numerical computations we let $L=N / 2$.

Setting up the discrete linear complementarity problem. We see now that the entries of the tridiagonal matrix (the implicit part) for the Lax-Wendroff scheme are given by

$$
\begin{aligned}
& a=-P k / h^{2}-\left(\tau+\tau^{2}\right) / 2 \\
& b=1+r k+2 k P / h^{2}+\tau^{2} \\
& c=-P k / h^{2}+\left(\tau-\tau^{2}\right) / 2
\end{aligned}
$$

with the quantities

$$
P:=\bar{\sigma}^{2} / 2, \quad Q=q-r+\bar{\sigma}^{2} / 2-\omega(h), \quad \mu=k / h, \quad \tau=\mu Q
$$

The entries for the central scheme are almost the same: We just drop the $\tau^{2}$-term wherever it appears. The resulting tridiagonal matrix whose entries are $a, b$ and $c$ is constant along its diagonals:

$$
T=\left[\begin{array}{ccccc}
b & c & & & \\
a & b & c & & \\
& \ddots & \ddots & \ddots & \\
& & a & b & c \\
& & & a & b
\end{array}\right]
$$


Using (45)-(48), the discretization for the right-hand side reads

$$
d_{n}^{m}=u_{n}^{m}+k h \sum_{l=-N / 2}^{N / 2}\left(u_{n+l}-u_{n}\right) k_{l} \rho_{l}
$$

Note that this vector needs to be updated for the boundary condition. For American call options this is done by updating the first and the last entries in $d^{m}$, i.e.

$$
d_{1}^{m} \leftarrow 0, d_{N-1}^{m} \leftarrow d_{N-1}^{m}-c\left(e^{x_{\max }}-K\right)
$$

Summarizing, the problems we wish to solve for $m=0,1, \ldots, M-1$, have the following form:

$$
\left\{\begin{array}{l}
T u^{m+1} \geq d^{m} \\
u^{m+1} \geq \psi \\
\left(T u^{m+1}-d^{m}, u^{m+1}-\psi\right)=0
\end{array}\right.
$$

Matrix $T$ is defined by (49)-(53), $d^{m}$ is given by the expression (54) with the update (55) and $\psi$ is the vector $\left[\psi_{1}, \psi_{2}, \ldots, \psi_{N-1}\right]^{T}$, with $\psi_{n}=\psi\left(x_{n}\right)$.

Because of the particular form of the problem and of matrix $T$, the BrennanSchwartz algorithm may be used to find the solution to this LCP. This will be explained next. The quantities $J_{n}$ appearing in $d^{m}$ will be treated separately.

\section{The Brennan-Schwartz Algorithm}

The well-known Brennan-Schwartz algorithm was originally developed for American put options, for which a rigorous justification can be found in Jaillet et al. (1990). The algorithm needs to be adapted for handling American call options, as mentioned in Jaillet et al. (1990). The natural modification needed is a straightforward reordering of indices, as explained in this section.

Let a tridiagonal matrix

$$
T=\left[\begin{array}{ccccc}
b_{1} & c_{1} & & & \\
a_{2} & b_{2} & c_{2} & & \\
& \ddots & \ddots & \ddots & \\
& & a_{n-1} & b_{n-1} & c_{n-1} \\
& & & a_{n} & b_{n}
\end{array}\right]
$$

and vectors $d=\left[d_{1}, \ldots, d_{n}\right]^{T}$ and $\psi=\left[\psi_{1}, \ldots, \psi_{n}\right]^{T}$ be given. Consider the following problem: find a vector $u$ satisfying the system

$$
\left\{\begin{array}{l}
T u \geq d \\
u \geq \psi \\
(T u-d, u-\psi)=0
\end{array}\right.
$$

The following algorithm results for an American call: 
- Step 1: Compute recursively a vector $\tilde{b}$ as

$$
\begin{aligned}
& \widetilde{b}_{1}=b_{1} \\
& \widetilde{b}_{j}=b_{j}-a_{j} c_{j-1} / \widetilde{b}_{j-1}, \quad j=2, \ldots, n
\end{aligned}
$$

- Step 2: Compute recursively a vector $\tilde{d}$ as

$$
\begin{aligned}
& \widetilde{d}_{1}=d_{1} \\
& \tilde{d}_{j}=d_{j}-a_{j} \widetilde{d}_{j-1} / \widetilde{b}_{j-1}, \quad j=2, \ldots, n
\end{aligned}
$$

- Step 3: Compute $u$ backwards:

$$
\begin{aligned}
& u_{n}=\max \left[\tilde{d}_{n} / \tilde{b}_{n}, \psi_{n}\right] \\
& u_{j}=\max \left[\left(\tilde{d}_{j}-c_{j} u_{j+1}\right) / \widetilde{b}_{j}, \psi_{j}\right], j=n-1, n-2, \ldots, 1
\end{aligned}
$$

We apply this algorithm using the matrix $T$ given in (53). The algorithm for the put option is analogous to the above algorithm, but the numbering of the indices must be reversed (Jaillet et al., 1990).

Remark 3.2. A more general method by Cryer (1983) allows the solution of (58) with the only requirement that $T$ is an $M$-matrix. Cryer's algorithm may be used to tackle problems where the exercise boundary is not connected, as in American butterfly spread options. This algorithm only requires $O(n)$ operations.

The splitting proposed in (35)-(37) does not satisfy the sufficient conditions of the Brennan-Schwartz algorithm. If we want to comply with these conditions, the term containing the first derivative in $\mathcal{A}$ should be moved to $\mathcal{B}$ instead, with only a minor change in the entries of matrix $T$. However, the explicit treatment of this convection term will result in a more demanding stability condition. The algorithm used is, however, provably correct if $\bar{\sigma}>0$ and $h$ is sufficiently small.

Fast convolution by FFT. The fast Fourier transform is an algorithm that evaluates the discrete Fourier transform (DFT) of a vector $f=\left[f_{0}, f_{2} \ldots, f_{R-1}\right]$ in $O(R \log R)$ operations.

The discrete Fourier transform is defined as:

$$
F_{k}=\sum_{n=0}^{R-1} f_{n} e^{-i 2 \pi n k / R}, \quad k=0,1, \ldots, R
$$

One of the multiple applications of the DFT is in computing convolutions. Let us first introduce the concept of circulant convolution. Let $\left\{x_{m}\right\}$ and $\left\{y_{m}\right\}$ be two sequences with period $R$. The convolution sequence $z:=x * y$ is defined componentwise as

$$
z_{n}=\sum_{m=0}^{R-1} x_{m-n} y_{m}
$$


We use now FFT to compute the vector $\left[z_{0}, \ldots, z_{R-1}\right]$. The periodic structure of $x$ allows the derivation of the following simple relation:

$$
Z_{k}=X_{k} \cdot Y_{k}
$$

where $X, Y$ and $Z$ denote the discrete Fourier transform of the sequences $x, y$ and $z$, respectively. That is, DFT applied to the convolution sequence is equal to the product of the transforms of the original two sequences. The vector $\left[z_{0}, \ldots, z_{R-1}\right]$ may be recovered by means of the inverse discrete Fourier transform (IDFT):

$$
z_{n}=\frac{1}{R} \sum_{k=0}^{R-1} Z_{k} e^{i 2 \pi k n / R}, \quad n=0,1, \ldots, R
$$

In the language of matrices, a circulant convolution may be seen as the product of a circulant matrix times a vector. For example, let $R=3$, and use the periodicity $x_{k}=x_{k+R}$ to arrive at

$$
\left[\begin{array}{l}
z_{0} \\
z_{1} \\
z_{2}
\end{array}\right]=\left[\begin{array}{lll}
x_{0} & x_{1} & x_{2} \\
x_{2} & x_{0} & x_{1} \\
x_{1} & x_{2} & x_{0}
\end{array}\right]\left[\begin{array}{l}
y_{0} \\
y_{1} \\
y_{2}
\end{array}\right]
$$

A circulant matrix is thus a matrix in which each row is a 'circular' shift of the previous row.

We are interested in the correlation sequence (45), where the vector $w$ is not periodic. The associated matrix is a so-called Toeplitz matrix, which by definition is a matrix that is constant along diagonals. A circulant matrix is hence a particular type of Toeplitz matrix. The next idea is to embed a Toeplitz matrix into a circulant matrix. As an example, let $L=1$ and $N=2$, so that the matrix-vector notation for (45) reads

$$
\left[\begin{array}{lll}
w_{1} & w_{0} & w_{-1} \\
w_{2} & w_{1} & w_{0} \\
w_{3} & w_{2} & w_{1}
\end{array}\right]\left[\begin{array}{c}
k_{1} / 2 \\
k_{0} \\
k_{-1} / 2
\end{array}\right]
$$

The matrix above may be embedded in a circulant matrix $C$ of size 5 in the following way:

$$
C=\left[\begin{array}{ccc|cc}
w_{1} & w_{0} & w_{-1} & w_{3} & w_{2} \\
w_{2} & w_{1} & w_{0} & w_{-1} & w_{3} \\
w_{3} & w_{2} & w_{1} & w_{0} & w_{-1} \\
\hline w_{-1} & w_{3} & w_{2} & w_{1} & w_{0} \\
w_{0} & w_{-1} & w_{3} & w_{2} & w_{1}
\end{array}\right]
$$

(For computational efficiency of the FFT algorithm, it is advisable to use a circulant matrix whose size is a power of 2.) If we define the vector $\eta:=\left[k_{1} / 2, k_{0}, k_{-1} / 2,0,0\right]^{T}$, then the product (64) is the vector consisting of the first three elements in the product $\mathrm{C} \eta$. As explained before, the product of a circulant matrix and a vector may be done efficiently by applying the FFT algorithm. 
As a summary, following the ideas explained above, it is possible to compute the correlation (45), with $L=N / 2$, by 'embedding' the resulting matrix into a circulant matrix. The product of a circulant matrix and a vector is carried out in three FFT operations, namely, two DFT and one IDFT.

For further details on the computation of convolutions by FFT we refer to Van Loan (1992).

\section{Second-order Timestepping Method}

The timestepping method considered so far gives an error of the form $O\left(k+h^{2}\right)$. In order to have a method that is also second-order accurate in time, we propose an extrapolated variant of the well-known BDF2 scheme.

The second-order timestepping method reads

$$
\frac{\frac{3}{2} u^{m+1}-2 u^{m}+\frac{1}{2} u^{m-1}}{k}-\mathcal{A} u^{m+1}-\mathcal{B} \bar{u}^{m}=0
$$

For the sake of readability the complementarity conditions have been omitted. Here

$$
\bar{u}^{m}:=2 u^{m}-u^{m-1}
$$

as for an explicit BDF2 scheme (Hundsdorfer, 2001). That is, this scheme is a mixture of implicit and explicit BDF2, where the implicit part is as before, a differential operator, and the explicit part is the integral operator away from the singularity. The initial vectors $u^{0}$ and $u^{1}$ are the payoff function and one Euler step according to scheme (38).

\section{Stability Analysis}

In this section we carry out a von Neumann stability analysis. We first study Euler's scheme:

$$
\frac{u^{m+1}-u^{m}}{k}-\mathcal{A} u^{m+1}-\mathcal{B} u^{m}=0
$$

where operators $\mathcal{A}$ and $\mathcal{B}$ are defined in (35) and (37).

Element-wise, the Euler scheme reads

$$
\begin{aligned}
u_{n}^{m+1}- & u_{n}^{m}-\delta\left[u_{n+1}^{m+1}-2 u_{n}^{m+1}+u_{n-1}^{m+1}\right] \\
& +\mu \gamma\left[u_{n+1}^{m+1}-u_{n-1}^{m+1}\right]+r k u_{n}^{m+1}-k h \sum_{l=-N / 2}^{N / 2}\left(u_{n+l}^{m}-u_{n}^{m}\right) k_{l} \rho_{l}
\end{aligned}
$$

where

$$
\mu=k / h, \quad \delta=\bar{\sigma}^{2} k / 2 h^{2}, \quad \gamma=\left(q-r-\bar{\sigma}^{2} / 2-\omega(h)\right) / 2
$$

For linear schemes like (69), a useful shortcut to finding the amplification factor $g$ is to substitute

$$
u_{n}^{m}=g^{m} e^{i n h \xi}
$$


into the scheme and do the simplifications; for examples see Strikwerda (1989). After some manipulations one arrives at the following linear equation in $g$ :

$$
\left[1+r k+2 \delta+(\mu \gamma-\delta) e^{i h \xi}+(-\mu \gamma-\delta) e^{-i h \xi}\right] g-[1+k h \Phi(h, \xi)]=0
$$

Here, we introduce the function

$$
\Phi(h, \xi):=\sum_{l=-N / 2}^{N / 2}\left(e^{i l h \xi}-1\right) k_{l} \rho_{l}=\frac{1}{v} \sum_{l=-N / 2}^{N / 2}\left(e^{i l h \xi}-1\right) \frac{e^{-\lambda_{ \pm}|l| h}}{|l| h} \rho_{l}
$$

The symbol $\lambda_{ \pm}$is a short-hand notation indicating that we take $\lambda_{+}$or $\lambda_{-}$if $l$ is positive or negative, respectively. The amplification factor turns out to be

$$
g=g(h, \xi)=\frac{1+k h \Phi(h, \xi)}{1+r k+2 \delta \sin ^{2}(h \xi / 2)+2 i \mu \gamma \sin (h \xi)}
$$

We are looking for an estimate of the form $|g| \leqslant 1$. That is, we need to estimate

$$
|g|^{2}=\frac{(1+k h \operatorname{Re} \Phi)^{2}+(k h \operatorname{Im} \Phi)^{2}}{\left(1+r k+2 \delta \sin ^{2}(\theta / 2)\right)^{2}+4 \mu^{2} \gamma^{2} \sin ^{2} \theta}
$$

where $\theta:=h \xi, \theta \in[0,2 \pi]$. Let us study the function $\Phi$ defined in (72). We focus on the positive part of the sum (as the negative part goes similarly). Consider the sum

$$
S(h)=\sum_{l=1}^{N / 2}\left(e^{i l h \xi}-1\right) \frac{e^{-\lambda_{+} l h}}{l}
$$

We drop the coefficients $\rho_{l}$. Letting $h \rightarrow 0$, the imaginary part tends to the integral

$$
\int_{0}^{x_{\max }} \frac{\sin (x \xi)}{x} e^{-\lambda_{+} x} \mathrm{~d} x
$$

The improper integral (letting $x_{\max } \rightarrow \infty$ ) has the known value $\arctan \left(\frac{\xi}{\lambda_{+}}\right)$, which is bounded. However, the real part is unbounded for $h \rightarrow 0$. We may find a lower bound for the real part as follows:

$$
\operatorname{Re} S(h)=-\sum_{l=1}^{N / 2} \frac{2 \sin ^{2}(l \theta / 2) e^{-\lambda_{+} l h}}{l} \geq-2 \sum_{l=1}^{N / 2} \frac{e^{-\lambda_{+} l h}}{l}
$$

Denote by $f(h)$ the sum on the right-hand side of (77). To find an expression for this sum, one differentiates element-wise with respect to $h$ to obtain a geometric series. The resulting expression is integrated and one arrives at the identity

$$
f(h)=-\left(1-e^{-\lambda_{+} x_{\max }}\right) \log \left(1-e^{-\lambda_{+} h}\right)
$$

The integration constant is easily seen to be zero by letting $h \rightarrow \infty$. It remains to observe now that for $h$ small and $\lambda_{+}>1$, the logarithm in (78) is greater than $\log h$.

Returning to (73): in order to find a bound for the numerator, we look for time steps $k$ such that $1+k h \operatorname{Re} \Phi \geqslant 0$. From the discussion above it follows that this 
is satisfied if

$$
k \leq-\frac{C}{\log (h)}
$$

for a certain constant $C$. For numerical purposes, this is a very mild restriction on the time step, i.e. given a time step, only a very small $h$ will violate this condition.

We now use a second elementary inequality: $x^{2}+y^{2} \leqslant(|x|+|y|)^{2}$, which, applied to the numerator of $g$, gives

$$
|g|^{2} \leq \frac{(1+k h \operatorname{Re} \Phi+k h|\operatorname{Im} \phi|)^{2}}{(1+r k)^{2}}
$$

On the other hand, for $h$ small enough, $\operatorname{Re} \Phi+|\operatorname{Im} \phi| \leqslant 0$. Hence, for $k$ satisfying (79), we obtained the desired $|g| \leqslant 1 /(1+r k)$.

We present in Figure 1 a plot of the function $g(\theta)$ for different values of $h$, but with fixed $k$. Observe that only for $M=5$ and $N=15000$ are there points outside the unit circle. Employing the parameters given in Figure 1, the constant in (79) becomes $C=1 / 4\left(1-e^{-\lambda_{+} x_{\max }}\right)$. For example, this implies that for time steps smaller than 0.09 , 0.05 and 0.03 , for $N=100,1000$ and 15000 respectively, the numerical solution is stable.

Let us now briefly look at the extrapolated BDF2 scheme. In this case, the amplification factors are the roots of the following second-order amplification polynomial:

$$
g_{5} g^{2}+g_{4} g+g_{3}
$$

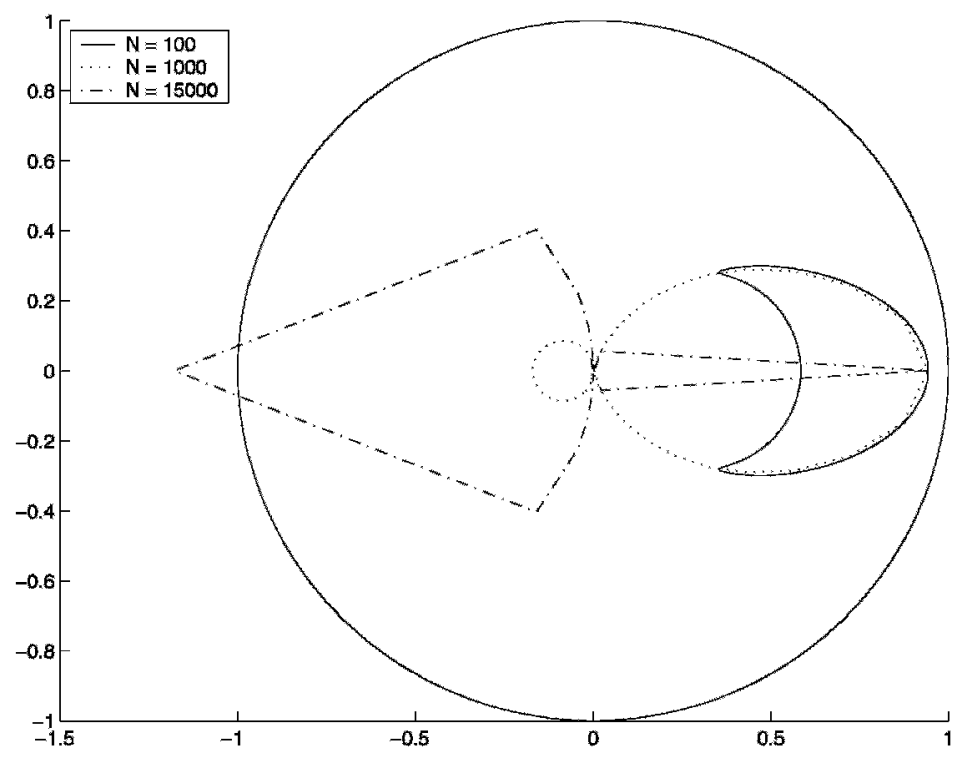

Figure 1. Amplification factor $g(\theta)$ for $M=5$ and different values of $N$. The parameters are: $r=0.3, q=0, \bar{\sigma}=0, K=1, T=1, v=1, \lambda_{+}=\lambda_{-}=5, x_{\text {min }}=-4, x_{\text {max }}=4$ 
where

$$
\begin{aligned}
& g_{5}=3 / 2+r k+2 \delta \sin ^{2}(\theta / 2)+2 i \mu \gamma \sin (\theta) \\
& g_{4}=-2(1+k h \Phi(h, \xi)) \\
& g_{3}=1 / 2+k h \Phi(h, \xi)
\end{aligned}
$$

The stability analysis for this case is more involved. We show in Figure 2 that the roots are different and that a more demanding time-step restriction is needed, as expected. For $N=2000$ we see that some of the roots fall outside the unit circle.

\section{Numerical Examples}

First, we carry out a reference experiment where we compute a European call option with Euler's timestepping and the central scheme. The parameters are:

$$
r=0.1, q=0, \lambda_{+}=5, \lambda_{-}=5, v=1, K=1, T=1
$$

Table 1 shows that the Euler scheme gives first-order convergence as expected. Note that the CPU times correspond to linear complexity in accordance with the FFT complexity.

In the second experiment we consider a put option with the same parameters (82). (Recall that the Brennan-Schwartz algorithm for a put is different from that for a call.) We show numerically that the implicit-explicit BDF2 scheme (66) is secondorder accurate in space and in time by comparing the numerical solution with a reference value computed with many grid points. The results are summarized in Table 2 .

Let us focus now on the smooth fit. The third experiment consists in computing the American call price using the following parameters:

$$
\sigma=0.2, \theta=0.085, v=1, K=1, N=2500 \text { and } M=500
$$
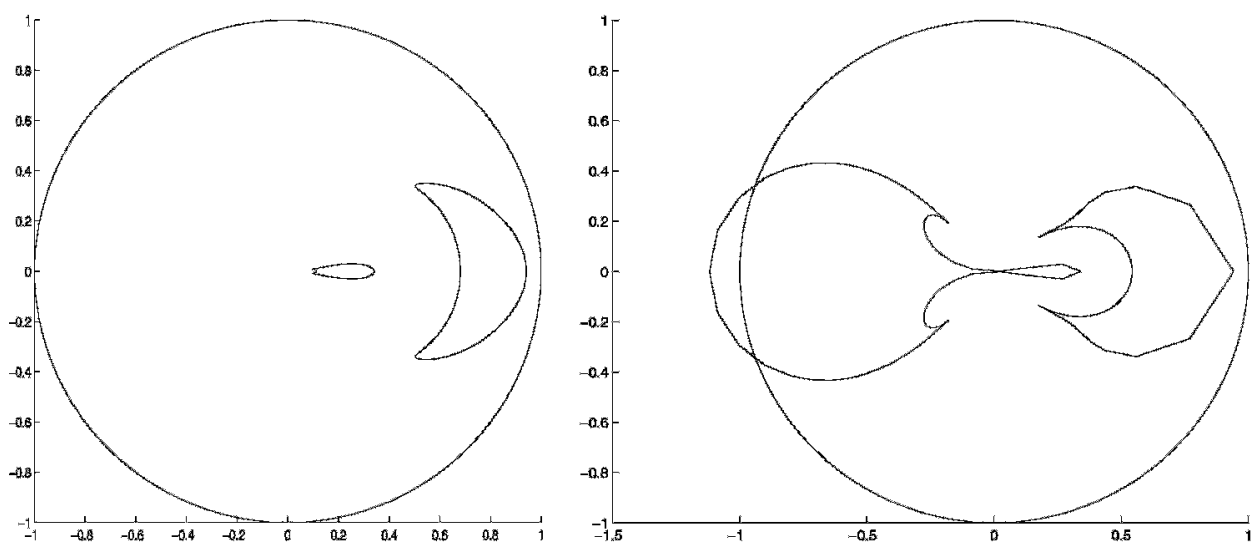

Figure 2. Amplification factor for the explicit BDF2 scheme with parameters as in Fig.1. Left: $N=100$; Right: $N=2000$ 
Table 1. First-order convergence for a VG European call

\begin{tabular}{lccc}
\hline$N$ & $M$ & Error at $x=0$ & CPU time (s) \\
\hline 100 & 10 & $5.2 \mathrm{E}-3$ & 0.02 \\
200 & 20 & $2.7 \mathrm{E}-3$ & 0.03 \\
400 & 40 & $1.3 \mathrm{E}-3$ & 0.06 \\
800 & 80 & $6.7 \mathrm{E}-4$ & 0.18 \\
1600 & 160 & $3.1 \mathrm{E}-4$ & 0.93 \\
Ref. value & & 0.15131 & \\
\hline
\end{tabular}

Table 2. Second-order convergence for a VG European put

\begin{tabular}{lcccc}
\hline$N$ & $M$ & error at $x=0$ & rate & CPU time (s) \\
\hline 100 & 10 & $1.7 \mathrm{E}-3$ & & 0.006 \\
200 & 20 & $4.1 \mathrm{E}-3$ & 4.1 & 0.01 \\
400 & 40 & $9.1 \mathrm{E}-5$ & 4.5 & 0.04 \\
800 & 80 & $2.5 \mathrm{E}-5$ & 3.6 & 0.15 \\
1600 & 160 & $6.5 \mathrm{E}-6$ & 3.8 & 0.87 \\
Ref. value & & 0.0640837 & & \\
\hline
\end{tabular}

Notice that $\sigma$ is needed in (8), (10). It is assumed first that the condition $q>r+\omega$ is satisfied $(\omega=-0.11)$ so let $r=0.1$ and $q=0.1$. In this case the sufficient condition $q>r+\omega_{-}$does not hold since $\omega_{-}=0.1$. Observe in Figure 3 that there is no smooth fit. This was already pointed out in Matache (2003). If we satisfy condition (17) by taking for example $q=0.3$, the lack of smooth fit becomes more obvious, as
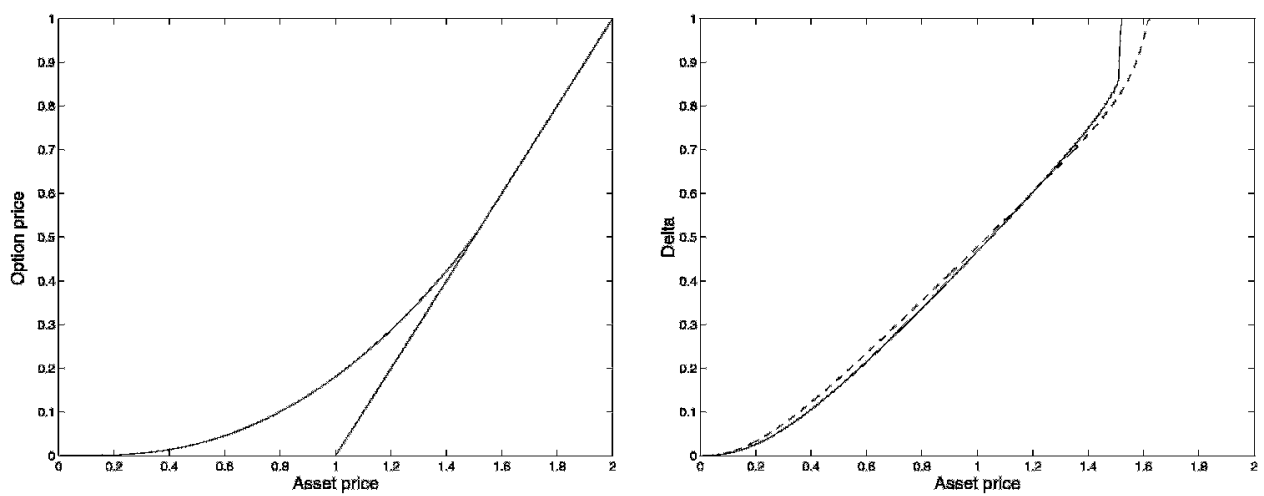

Figure 3. Left: VG option value and payoff function; Right: Delta for $\bar{\sigma}=0.1$ (dashed line) and for $\bar{\sigma}=0$ (continuous line). The parameters are: $r=0.1, q=0.1, \sigma=0.2, \theta=0.085, v=1$, $K=1, N=2500, M=500$ and $T=9$ 

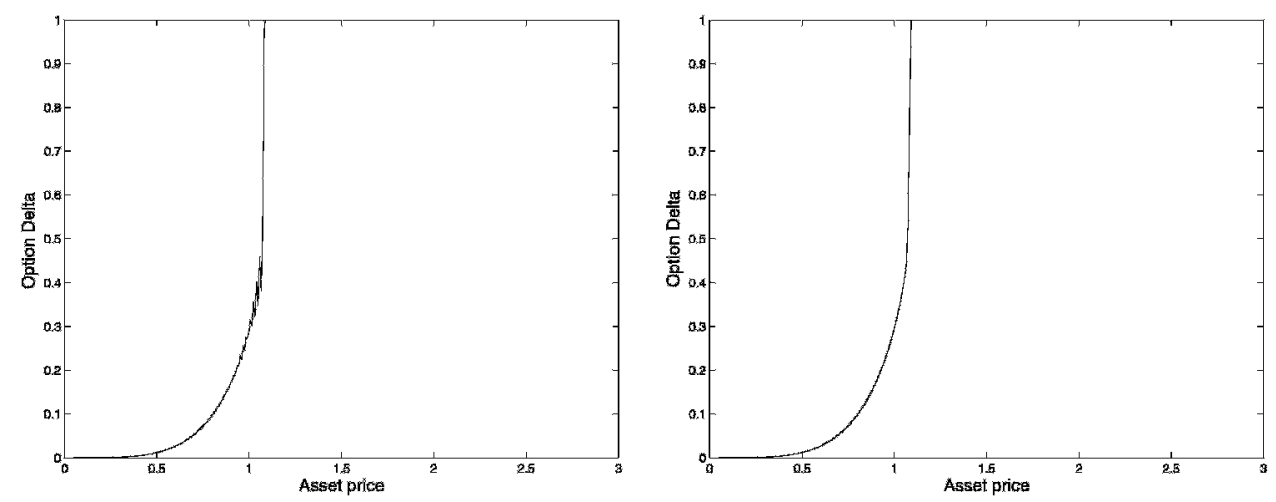

Figure 4. Left: Wiggles close to jump in the Delta due to central discretization. Right: The wiggles disappear with the Lax-Wendroff discretization

illustrated in Figure 4. This picture shows that the numerical Delta has small oscillations close to the jump. To avoid these oscillations, we propose using the LaxWendroff scheme for the convection term. The result of this choice is displayed in Figure 4 (right picture). The same applies to a put option with for example $r=0.3$ and $q=0$.

Figure 3 (right) also displays the smearing effect on the continuity of the option Delta after including a small diffusion $\bar{\sigma}$ in the VG model. For $\bar{\sigma}=0$ the figure shows that the smearing does not appear. Related theoretical results for finite activity processes may be found in the work of Pham (1997).

Next, we examine the behaviour of the free boundary near expiry. Two cases are distinguished: (a) $q \leqslant r+\omega_{-}$and $(b) q>r+\omega_{-}$. In the first case we let $r=0.1$ and $q=0.1$ and find the asymptotic behaviour of the free boundary to be $c(k)=1.1246$ (see
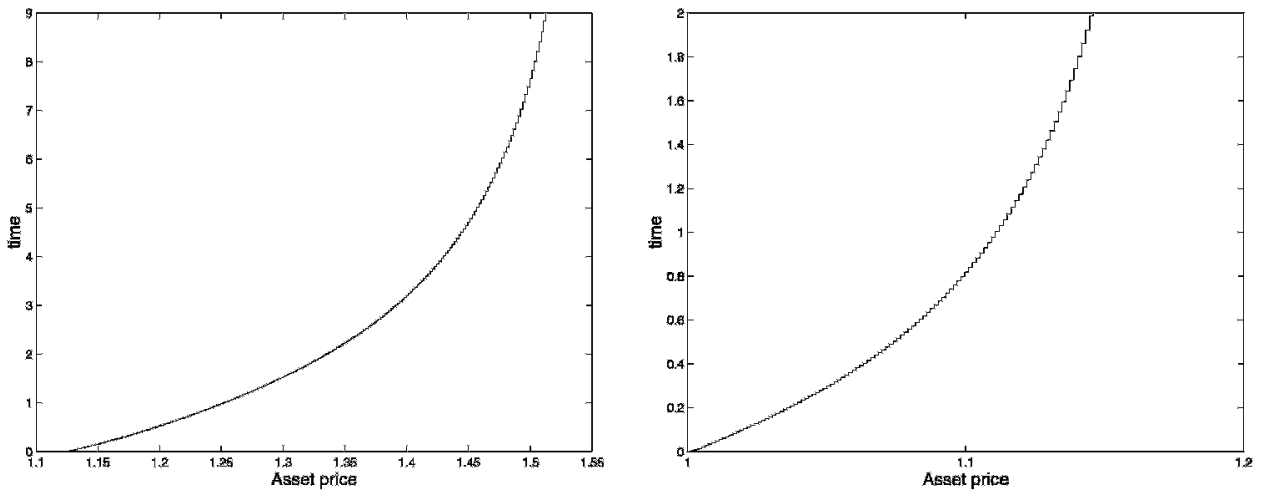

Figure 5. Left: Free boundary when $q<r+\omega_{-}$; parameters: $T=9, r=0.1, q=0.1, \sigma=0.2$, $\theta=0.085, v=1, K=1, M=2000$ and number of spatial points $N=8000$. Right: Free boundary when $q>r+\omega_{-}$; parameters: $T=2, r=0.1, q=0.21$ and the other parameters are the same as in the left picture 
Table 3. Stability tests for a call option with parameters $M=5, r=0.1, q=0, \lambda_{+}=5, \lambda_{-}=5, v=1$, $K=1, T=1$

\begin{tabular}{lcc}
\hline$N$ & BDF2 & Euler \\
\hline 100 & $\mathrm{v}$ & $\mathrm{v}$ \\
1000 & $\mathrm{v}$ & $\mathrm{v}$ \\
3000 & $*$ & $\mathrm{v}$ \\
10000 & $*$ & $\mathrm{v}$ \\
\hline
\end{tabular}

Figure 5, left). It is not difficult to verify that $\zeta(1.1246) \approx 0$, where

$$
\zeta(s)=q s-r K-\int_{-\infty}^{\ln (K / s)}\left(K-s e^{y}\right) k(y) \mathrm{d} y, \quad s>0
$$

In the second situation we choose $r=0.1$ and $q=0.22$, since $\omega_{-}=0.1$. Figure 5 (right) shows that $c(k) \approx 1=K$.

Finally, we summarize in Table 3 some numerical stability test results for both the Euler scheme and the extrapolated BDF2 using the central scheme for convection, computing a call option. The parameters are as in Figures 1 and 2; in particular the number of time steps is set to $M=5$, and the number of spatial steps $N$ varies. The ' $\mathrm{v}$ ' and ' $*$ ' in the table symbolize observed stable and unstable behaviour, respectively. The stable solutions are oscillation free, whereas the unstable ones are not. As we see, the results are in accordance with the stability analysis. In particular, we noticed that the Euler scheme behaves somewhat better than predicted by the theory.

\section{Conclusions}

In the first part of this paper it was made plausible that the smooth fit principle fails for the VG American call. The failure of the principle had already been pointed out in the financial literature for a large family of pure-jump processes in the case of infinite horizon. In the second part we proposed a numerical method to deal with the American call. This method is easy to implement and may be used for general Lévy processes with finite activity, even when the Lévy measure is obtained from calibration. The method does not require knowledge of the characteristic function to find the European price, as in, e.g., (Carr and Madan, 1999).

\section{Acknowledgements}

This research was supported by the Dutch government through the national program BSIK: knowledge and research capacity, in the ICT project BRICKS (http://www.bsik-bricks.nl), theme MSV1.

We would like to thank the referees for valuable comments.

\section{References}

Alili, L. and Kyprianou, A. E. (2005) Some remarks on first passage of Lévy processes, the American put and pasting principles, Annals Applied Probability, 15, pp. 2062-2080. 
Almendral, A. (2005) Numerical valuation of American options under the CGMY process, in: W. Schoutens et al. (Eds), Exotic Option Pricing and Advanced Lévy Models (Chichester: Wiley).

Almendral, A. and Oosterlee, C. W. (2005) Numerical valuation of options with jumps in the underlying, Applied Numerical Mathematics, 53, pp. 1-18.

Andersen, L. and Andreasen, J. (2000) Jump-diffusion processes: volatility smile fitting and numerical methods for option pricing, Review of Derivatives Research, 4, pp. 231-262.

Bensoussan, A. and Lions, J.-L. (1984) Impulse Control and Quasivariational Inequalities, $\mu$ (Montrouge: Gauthier-Villars).

Boyarchenko, S. I. and Levendorskiü, S. Z. (2002) Non-Gaussian Merton-Black-Scholes Theory, Vol. 9 of Advanced Series on Statistical Science \& Applied Probability (River Edge, NJ: World Scientific Publishing Co. Inc.).

Carr, P. P. and Madan, D. B. (1999) Option valuation using the Fast Fourier Transform, Journal of Computational Finance, 2, pp. 61-73.

Carr, P. P. et al. (1998) The Variance Gamma process and option pricing, European Finance Review, 2, pp. 79-105.

Cont, R. and Tankov, P. (2004) Financial Modelling with Jump Processes (Boca Raton, FL: Chapman \& Hall).

Cont, R. and Voltchkova, E. (2005) A finite difference scheme for option pricing in jump diffusion and exponential Lévy models, SIAM Journal Numerical Analysis, 43, pp. 1596-1626.

Cryer, C. W. (1983) The efficient solution of linear complementarity problems for tridiagonal Minkowski matrices, ACM Transactions Mathematics Software, 9, pp. 199-214.

D'Halluin, Y. et al. (2004) A penalty method for American options with jump diffusion processes, Numered Mathematics, 97, pp. 321-352.

D'Halluin, Y. et al. (2005) Robust numerical methods for contigent claims under jump diffusion processes, IMA Journal Numbered Analysis, 25, pp. 62-92.

Hirsa, A. and Madan, D. B. (2004) Pricing American options under variance gamma, Journal of Computational Finance, 7.

Hundsdorfer, W. (2001) Partially implicit BDF2 blends for convection dominated flows, SIAM Journal Numbered Analysis, 38, pp. 1763-1783.

Jaillet, P. et al. (1990) Variational inequalities and the pricing of American options, Acta Applied Mathematics, 21, pp. 263-289.

Karatzas, I. and Shreve, S. E. (1998) Methods of Mathematical Finance, Vol. 39 of Applications of Mathematics (New York: Springer-Verlag).

Koponen, I. (1995) Analytic approach to the problem of convergence of truncated Lévy flights towards the Gaussian stochastic process, Physical Review E, 52, pp. 1197-1199.

LeVeque, R. J. (1990) Numerical Methods for Conservation Laws, Lectures in Mathematics ETH Zürich, Birkhäuser Verlag, Basel.

Madan, D. B. and Seneta, E. (1990) The variance gamma (V.G.) model for share market returns, Journal of Business, 63, pp. 511-524.

Matache, A. M. et al. (2003) Wavelet Galerkin pricing of American options on Lévy driven assets. Working paper, ETH, Zürich.

Pham, H. (1997) Optimal stopping, free boundary, and American option in a jump-diffusion model, Applied Mathematics Optimisation, 35, pp. 145-164.

Samuelson, P. A. (1965) Rational theory of warrant pricing, Industrial Management Review, 6, pp. 13-31.

Strikwerda, J. C. (1989) Finite Difference Schemes and Partial Differential Equations, The Wadsworth \& Brooks/Cole Mathematics Series, Wadsworth \& Brooks/Cole Advanced Books \& Software, Pacific Grove, CA.

Van Loan, C. (1992) Computational Frameworks for the Fast Fourier Transform, Vol. 10 of Frontiers in Applied Mathematics, Society for Industrial and Applied Mathematics (SIAM), Philadelphia, PA.

Zhang, X. L. (1997) Numerical analysis of American option pricing in a jump diffusion model, Mathematics Operational Research, 22, pp. 668-690. 